\title{
Quantum-information processing in disordered and complex quantum systems
}

\author{
Aditi Sen(De), ${ }^{1,2}$ Ujjwal Sen, ${ }^{1,2}$ Veronica Ahufinger, ${ }^{3}$ Hans J. Briegel, ${ }^{4,5}$ Anna Sanpera, ${ }^{2,6}$ and Maciej Lewenstein ${ }^{2,7}$ \\ ${ }^{1}$ ICFO-Institut de Ciències Fotòniques, Parc Mediterrani de la Tecnologia, E-08860 Castelldefels (Barcelona), Spain \\ ${ }^{2}$ Institut für Theoretische Physik, Universität Hannover, D-30167 Hannover, Germany \\ ${ }^{3}$ ICREA and Grup d'Optica, Universitat Autònoma de Barcelona, E-08193 Bellaterra, Spain \\ ${ }^{4}$ Institut für Quantenoptik und Quanteninformation, Österreichische Akademie der Wissenschaften, A-6020 Innsbruck, Austria \\ ${ }^{5}$ Institut für Theoretische Physik, Universität Innsbruck, Technikerstrasse 25, A-6020 Innsbruck, Austria \\ ${ }^{6}$ ICREA and Grup de Física Teòrica, Universitat Autònoma de Barcelona, E-08193 Bellaterra, Spain \\ ${ }^{7}$ ICREA and ICFO-Institut de Ciències Fotòniques, Parc Mediterrani de la Tecnologia, E-08860 Castelldefels (Barcelona), Spain
}

(Received 15 October 2005; published 18 December 2006)

\begin{abstract}
We study quantum information processing in complex disordered many body systems that can be implemented by using lattices of ultracold atomic gases and trapped ions. We demonstrate, first in the short range case, the generation of entanglement and the local realization of quantum gates in a disordered magnetic model describing a quantum spin glass. We show that in this case it is possible to achieve fidelities of quantum gates higher than in the classical case. Complex systems with long range interactions, such as ions chains or dipolar atomic gases, can be used to model neural network Hamiltonians. For such systems, where both long range interactions and disorder appear, it is possible to generate long range bipartite entanglement. We provide an efficient analytical method to calculate the time evolution of a given initial state, which in turn allows us to calculate its quantum correlations.
\end{abstract}

DOI: 10.1103/PhysRevA.74.062309

PACS number(s): 03.67.Mn, 03.67.Lx, 05.50.+q, 05.30.Jp

\section{INTRODUCTION}

Successful implementations of quantum information processing (QIP) in atomic, molecular, or solid state systems typically demand a very rigorous control of the parameters governing the dynamics of the system [1]. This concern affects systems with few qubits such as the Cirac-Zoller computer [2] with ions or photons [3], as well as atomic gases in optical lattices [4]. Despite the recent progress, the demanded control in such systems is currently very hard to achieve [5]. Recently QIP in systems with a limited knowledge of the parameters has also been proposed [6].

Here we analyze QIP in systems with quenched (i.e., immobile in the dynamic scale) disorder. This may sound like contradicto in adjecto. However, in our system, disorder is not due to uncontrolled values of some of the system parameters. It has been recently shown that it is possible to create controlled disorder in atomic gases in optical lattices, and this opens up the possibility to study, in an unconventional way, Anderson and Bose glasses in a Bose gas [7], or spin glasses with short range interactions in Fermi-Bose, or BoseBose mixtures [8]. Disordered systems exist in nature, and the consequences of disorder are largely recognized, but they are still far from being well-understood [9]. We want here to deepen our understanding on the effects of quenched disorder for QIP in systems that can be created with currently available technology. For instance, using linear chains of trapped ions [10], or dipolar atomic gases [11], it is possible to realize complex spin systems with long range interactions that may serve as a model for classical and quantum neural networks [12] (cf. [13]).

We aim at using many body complex disordered systems to perform distributed QIP. However, before that, one should address more fundamental questions, namely (i) Can one generate entanglement in such systems that would survive quenched averaging over long times?

(ii) Can one realize quantum gates with reasonable fidelity in disordered systems?

Here we answer both questions affirmatively considering both short and long range disordered systems.

The reason for considering disordered systems for QIP is that potentially they could offer advantages for quantum information tasks as compared to ordered systems. First, since they have typically a large number of different metastable (free) energy minima, such states might be used to store information distributed over the whole system, similarly to neural network models [14]. The information is thus naturally stored in a redundant way, like in error correcting schemes [15]. Second, in disordered systems with long range interactions, the stored information is robust: metastable states have quite large basins of attraction in the thermodynamical sense.

In this paper, we first consider a short range disordered Ising Hamiltonian, the so-called Edwards-Anderson (EA) model of spin glass (SG) which can be straightforwardly implemented using atomic Bose-Fermi or Bose-Bose mixtures in optical lattices $[8,16]$. We address the generation and evolution of nearest neighbor $(\mathrm{NN})$ entanglement in this model. In the short range Ising model without disorder, it is possible to create cluster and graph states (and therefore, entanglement) starting from an appropriate initial product state $[17,18]$. Here we show that, while the density matrix of two neighboring spins averaged over the disorder remains always separable, the disorder averaged entanglement (quantified by logarithmic negativity [19]) converges in time to a finite value. We also show that the quantum single-qubit Hadamard gate can be realized in such a system with significant fidelity.

Second, we consider complex systems with long range $\left(1 / r^{3}\right.$ or $\left.1 / r^{2}\right)$ interactions that can be realized, for instance, 
in linear ion traps, using either local magnetic fields, as proposed by Wunderlich and co-workers [20], or by appropriately designed laser excitations [10]. The corresponding Hamiltonian can be mapped into an Ising neural network model with weighted patterns [14]. One can also include external parallel or transverse fields in the model. In such a system, we demonstrate analytically that it is possible to generate long range bipartite entanglement that undergoes a series of collapses and revivals [21]. Finally we also address the dynamics of bipartite and tripartite entanglement in an infinite range Ising model without disorder.

The paper is organized as follows. We begin in Sec. II by a definition of quenched averaging that is used in the physics of disordered systems [9]. This is followed in Sec. III by a definition of logarithmic negativity, the measure of entanglement that we use in this paper. In Sec. IV, we show that there is bipartite nearest neighbor entanglement that survives quenched averaging in short range disordered models. In the different sections of Sec. IV, we discuss the dependence of nearest neighbor entanglement on the time of evolution, on the lattice geometry, and on the choice of the initial state for the evolution. Section V is devoted to the implementation of quantum gates in disordered short range interaction systems. We then move to the case of long range interaction Hamiltonians (Sec. VI). We present first an analytical method to calculate bipartite as well as multipartite density matrices for long range interactions. We discuss the ordered and disordered cases separately. Curiously, both cases present revivals and collapses of bipartite entanglement. We summarize our results in Sec. VII.

\section{QUENCHED AVERAGING}

In the disordered systems that we consider in this paper, the disorders are "quenched," i.e., they remain unchanged at least for the duration of the considered dynamics of the system. In the systems that we consider, it is the interactions that are disordered. They are random variables following certain probability distributions. The disorder is quenched means that a particular realization of all the random variables remains fixed for the relevant duration of the dynamics of the system. Due to this reason, an average of a physical quantity, say $A$, is to be carried out in the following order.

(a) First we consider a particular realization of the system and take the corresponding fixed values of the system parameters (including the ones that are disordered).

(b) Then, we compute the value of the physical quantity $A$, with those fixed parameter values.

(c) Lastly, we average over the disordered parameters. This mode of averaging is called "quenched" averaging. It may be mentioned that an averaging in which items (b) and (c) are interchanged in order is called "annealed" averaging.

\section{LOGARITHMIC NEGATIVITY}

To study bipartite entanglement, we use the logarithmic negativity (LN) [19]. The LN of a bipartite state $\rho_{A B}$ is defined as

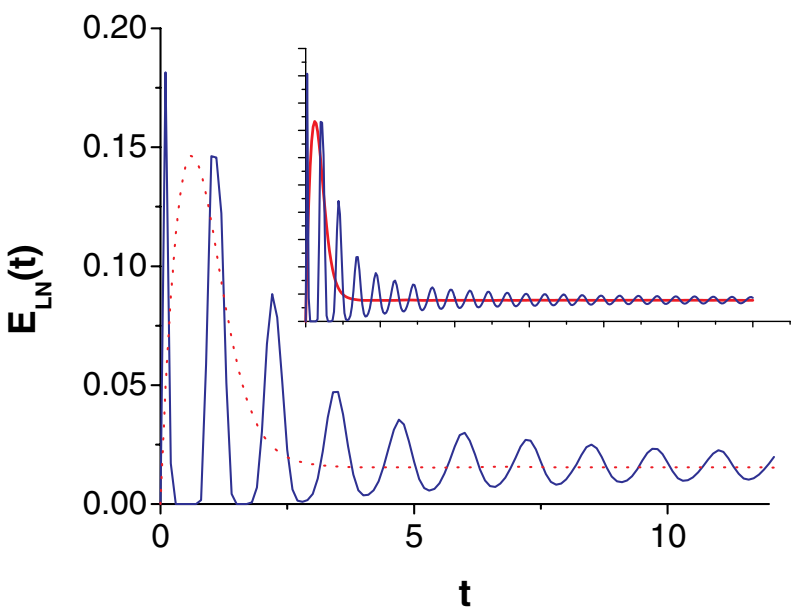

FIG. 1. (Color online) Temporal behavior of NN averaged entanglement in a 2D SG model, starting from $\Pi_{i}|+\rangle_{i}$. $E_{L N}(t)$ denotes the $\mathrm{NN}$ averaged logarithmic negativity and $t$ denotes the time. (Note that $t$ is implicitly multiplied by $c / \hbar$, so that the horizontal axis is actually of $c t / \hbar$.) For a model with frustration $(J=0), E_{L N}(t)$ converges quickly to a constant value (red dotted curve). For a nonfrustrated case $(J=+5), E_{L N}(t)$ exhibits damped oscillations (blue curve), converging to the same value $\approx 0.0154$, as reached in the frustrated case. Standard deviation for $t \rightarrow \infty$ is $\approx 0.0704$. It is interesting to note that the dynamical behavior of $E_{L N}$ depends on $J$, although at large times, they all converge to the same value (see inset). The same behavior is encountered in the $1 \mathrm{D}$ case, even though there is no frustration in that case.

$$
E_{L N}\left(\rho_{A B}\right)=\log _{2}\left\|\rho_{A B}^{T_{A}}\right\|_{1},
$$

where $\|\cdot\|_{1}$ is the trace norm, and $\rho_{A B}^{T_{A}}$ denotes the partial transpose of $\rho_{A B}$ with respect to the $A$-part [22]. Note that $\rho_{i j}(t)$ acts on $\mathrm{C}^{2} \otimes \mathrm{C}^{2}$. Consequently, a nonzero value of the LN implies that the state is entangled and distillable [22,23], while $E_{L N}=0$ implies separability [22]. The LN of the singlet state $(|01\rangle-|10\rangle) / \sqrt{2}$ is unity, which will serve as our unit of entanglement. (Throughout the paper, $|0\rangle$ and $|1\rangle$ are eigenvectors of the Pauli $\sigma^{z}$ operator, with eigenvalues +1 and -1 , respectively.) The singlet is said to have one ebit (entanglement bit).

\section{DYNAMICS OF ENTANGLEMENT WITH A SHORT- RANGE DISORDERED HAMILTONIAN}

\section{A. Entanglement of the evolved state of the Edwards- Anderson spin glass model: Quenched averaging}

The short range disordered Hamiltonian, the EdwardsAnderson SG model, that we consider here is given by

$$
H_{E-A}=-\frac{c}{4} \sum_{\langle i j\rangle} J_{i j} \sigma_{i}^{z} \sigma_{j}^{z} .
$$

Here $\sigma_{k}^{z}$ denotes the Pauli operator at the $k$ th site, and $J_{i j}$ 's describe NN couplings for an arbitrary lattice. The prefactor $c$ is used to make the couplings $J_{i j}$ dimensionless, and also to make them effectively of the order of unity. In the EA model 
these couplings are given by independent Gaussian variables with mean $J$ and variance $\sigma^{2}$. Starting from a pure product state of the form [18]

$$
|\Psi\rangle=\prod_{i}|+\rangle_{i}, \quad \text { where }| \pm\rangle=(|0\rangle \pm|1\rangle) / \sqrt{2},
$$

we evaluate the entanglement after a finite time. The density matrix is given by

$$
\begin{aligned}
\rho\left(t,\left\{J_{i j}\right\}\right) & =\exp \left\{-\frac{i}{\hbar} H_{E-A} t\right\}|\Psi\rangle\langle\Psi| \exp \left\{+\frac{i}{\hbar} H_{E-A} t\right\} \\
& =\exp \left\{-i \frac{1}{c} H_{E-A} \frac{c t}{\hbar}\right\}|\Psi\rangle\langle\Psi| \exp \left\{+i \frac{1}{c} H_{E-A} \frac{c t}{\hbar}\right\}
\end{aligned}
$$

and the reduced density matrix for any NN pair is obtained by tracing over all other sites. (In the following, $t$ will mean $c t / \hbar$, i.e., it will always be implicitly multiplied by a factor $c / \hbar$.) For example, the reduced density matrix for a twodimensional (2D) square lattice of the pair $(1,2)$ is given by

$$
\begin{aligned}
\varrho_{12}\left(t,\left\{J_{i j}\right\}\right)= & \frac{1}{4} \otimes \mathbf{1}+\frac{1}{4}\left[\cos \left(J_{24} t / 2\right) \cos \left(J_{26} t / 2\right) \cos \left(J_{28} t / 2\right)\right. \\
& \times\left\{e^{i J_{12} t / 2}|00\rangle\left\langle 01\left|+e^{-i J_{12} t / 2}\right| 10\right\rangle\langle 11|\right\} \\
& +\cos \left(J_{13} t / 2\right) \cos \left(J_{15} t / 2\right) \cos \left(J_{17} t / 2\right)\left\{e^{i J_{12} t / 2}|00\rangle\right. \\
& \left.\times\left\langle 10\left|+e^{-i J_{12} t / 2}\right| 01\right\rangle\langle 11|\right\} \\
& +\cos \left(J_{13} t / 2\right) \cos \left(J_{15} t / 2\right) \cos \left(J_{17} t / 2\right) \\
& \times \cos \left(J_{24} t / 2\right) \cos \left(J_{26} t / 2\right) \cos \left(J_{28} t / 2\right)\{|00\rangle\langle 11| \\
& +|01\rangle\langle 10|\}+ \text { H.c. }],
\end{aligned}
$$

where $\mathbf{1}$ is the identity operator on a two-dimensional complex Hilbert space, and the indices $3,4, \ldots, 8$ enumerate the six neighbors of 1 and 2. [We assume that either the lattice is infinite, or that none of the $(1,2)$ pair is at the boundary of the lattice.] As we have mentioned before, in the physics of disordered systems, to determine the values of relevant physical quantities such as free energy, entanglement, etc., we are obliged to perform a quenched average [9] (see also $[24,25])$. The disorder averaged state is actually separable. So, the annealed averaged entanglement is zero (see Sec. II).

The entanglement in the SG model turns out to be an even function of the couplings. The temporal behavior of $E_{L N}(t)$ in a 2D square lattice is shown in Fig. 1 for two different cases of disorder: with frustration and without it. For $J=0, \sigma^{2}=1$, the system has randomly ferromagnetic $(J>0)$ and antiferromagnetic $(J<0)$ interactions, and is strongly frustrated; $E_{L N}(t)$ is rapidly damped to a constant and does not show any oscillations. This behavior differs from the nonfrustrated case $J=5, \sigma^{2}=1$, when $E_{L N}(t)$ exhibits oscillations with frequencies $\sim \frac{J}{5} \frac{c}{\hbar}$. To understand why entanglement converges in time to the same finite value for both the frustated and nonfrustated cases, notice that as long as the distributions of the $J_{i j}$ 's are sufficiently well-behaved, $J_{i j} t / 2$ corresponds to a uniform distribution over $[0,2 \pi]$ for large enough $t[26]$, and the sign of $J_{i j}[(i, j) \neq(1,2)]$ does not play any role.
Note here that for short range interactions, the nextnearest neighbor entanglement vanishes, even before the averaging, for both $1 \mathrm{D}$ and $2 \mathrm{D}$ square lattices.

\section{B. Time dependence of the nearest neighbor entanglement: Probability distributions at given times}

From the previous calculations we have seen that for a 2D square lattice, the quenched averaged $\mathrm{NN}$ entanglement converges, for large times, to a very low value. Precisely, $E_{L N}$ $=0.0154$. To better understand the time dependence of the above results we analyze here the probability distribution of entanglement at given times, for the same lattice geometry (viz. 2D square lattice). The probability distributions are obtained by considering the relative frequencies of the entanglements for a given distribution of the $J_{i j}$ 's, and is calculated before the quenched averaging.

The probability distributions at two exemplary times are plotted in the histograms in Fig. 2. They show that although the average value of $\mathrm{NN}$ entanglement is small, there is always a finite probability of obtaining an entanglement that is one order of magnitude larger than the quenched averaged value. For example, we observe that at small times, the probability of obtaining bipartite entanglement between the values 0.2 and 0.4 is about $15 \%$. Intuitively, this is the entanglement that is useful in obtaining the nonclassical fidelities for the quantum gate operation considered in Sec. V.

Both short- and long-time behaviors of the entanglement are considered in Fig. 2 by looking at their probability distribution at given times. We observe that there is a marked difference between short- and long-time behaviors. While the probability decays exponentially with increasing entanglement at large times, it is not so for small times (see Fig. 3).

\section{Lattice geometry dependence of the nearest neighbor entanglement}

To study the dependence of the NN bipartite entanglement with the lattice geometry we have studied the above problem for the following lattice configurations: linear chain, 2D honeycomb lattice, 2D square, and 3D cube. Any given pair of neighboring lattice sites in the above configurations have $d$ $=2,4,6$, and 10 neighbors, respectively. Our numerical simulations reveal that the NN entanglement also decays exponentially with increasing of neighbors for large enough times.

We can provide an analytical estimate that reproduces such dependence on the number of neighbors by considering the volume of the set of separable states (see, e.g., [28]) in a two-qubit system. This gives an upper bound on the NN entanglement that depends exponentially on $d$. To see this, consider an arbitrary lattice configuration, in which there are $d$ neighbors for any given pair of neighboring lattice sites. If the reduced density matrix between two neighboring lattice sites, $\rho_{i j}^{d}(t)$, is entangled, then we must necessarily have

$$
\left\|\frac{1}{4} \mathbf{1} \otimes \mathbf{1}-\rho_{i j}^{d}(t)\right\|_{2}>R,
$$

with $R$ being the radius of the separable ball in the two qubit space, using Frobenius norm (or 2-norm), where the latter is 


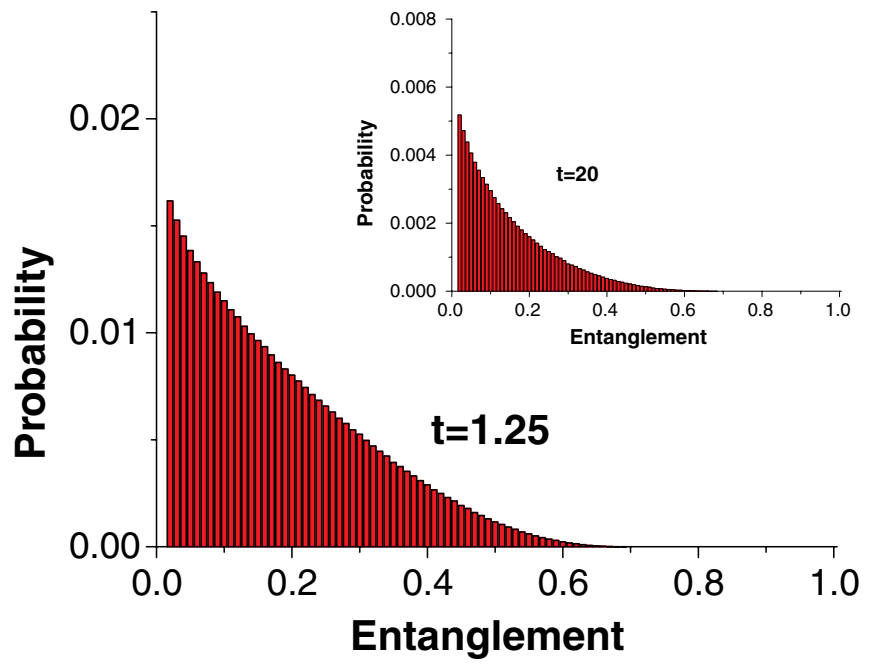

FIG. 2. (Color online) Probability distribution of NN entanglement for given times $t$ in the evolved state $\varrho_{12}\left(t,\left\{J_{i j}\right\}\right)$ of the Edwards-Anderson spin glass model [27]. The value of entanglement for a large number of sample $J_{i j}$ points (and hence a large probability) is concentrated in the "zero" entanglement zone $[0,0.01]$. This probability column is not shown in both of the histograms for easy viewing of the histograms. This large zero entanglement zone probability leads to the low average value of entanglement. However, the histograms show that there is a substantial probability of obtaining entanglement that is one order of magnitude more than the average value (see text). Note that including the zero entanglement probability, the histograms are in fact correctly normalized.

defined as $\|A\|_{2}=\sqrt{\operatorname{tr}\left(A^{\dagger} A\right)}$. Using the approximation $\cos x$ $\approx 1-x^{2} / 2$, the inequality (4) reduces to

$$
\sum_{i=1}^{d} \phi_{i}^{2}<\left(3-4 R^{2}\right) / 2,
$$

where the $\phi_{k}=J_{i j} t / 2$ 's are state parameters varying from 0 to $2 \pi$ (as they always appear as arguments of trigonometric functions) [29]. The volume of this hypersphere [the one given by Eq. (5)] is

$$
\mathcal{V}_{d}=\mathcal{S}_{d}\left(\frac{3-4 R^{2}}{2}\right)^{d / 2} / d,
$$

where $\mathcal{S}_{d}=2 \pi^{d / 2} / \Gamma(d / 2)$. Due to the periodicity involved implicitly in $\rho_{i j}^{d}(t)$, there are $2^{d}-1$ such hyperspheres [30]. Considering all states in this volume to have unit entanglement, the average NN entanglement, of a lattice that has $d$ neighbors for any neighboring pair of lattice sites, at sufficiently long times, is bounded by

$$
\mathcal{E}_{d}=\mathcal{V}_{d}\left(2^{d}-1\right) /(2 \pi)^{d}
$$

As an example, for the case of the 2D square lattice (for which $d=6$ ), at long times, the actual [i.e., calculated using the exact state in Eq. (4)] entanglement is $\approx 0.0154$, while the above estimated upper bound is $\mathcal{E}_{6} \approx 0.0221$. Although bipartite entanglement vanishes with increasing number of

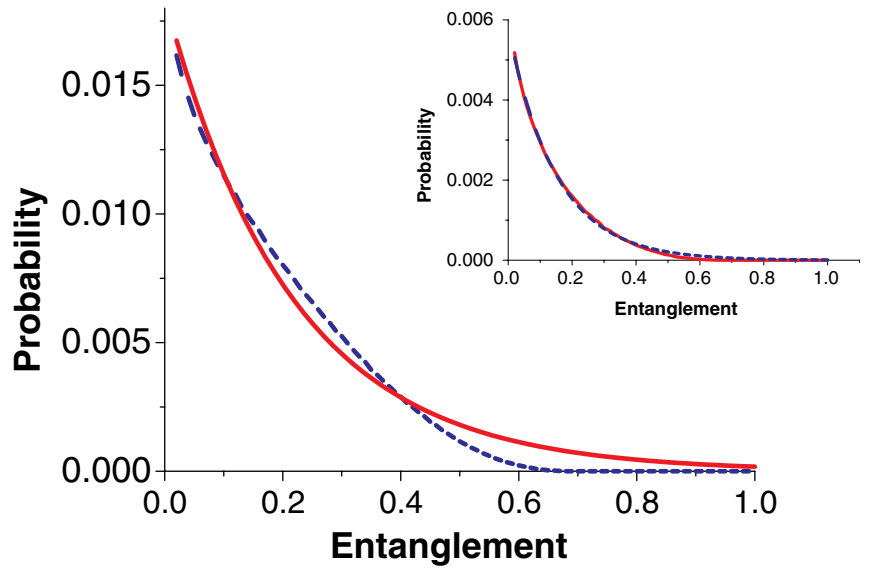

FIG. 3. (Color online) Plot of probability vs NN entanglement for time $t=1.25$ (and $t=20$ in inset) of $\varrho_{12}\left(t,\left\{J_{i j}\right\}\right)$ [27]. The probability for the entanglement range $[0.01 i, 0.01(i+1)]$ is plotted against an entanglement of $0.01 i$, for $i=1$ to 100 . The dashed (blue) lines correspond to the actual values, obtained by sampling a large number of $J_{i j}$ 's. The undashed (red) lines are exponential fits. The exponential fit in the inset (for $t=20$ ) is almost exact, while the one for $t=1.25$ is seen to not fit very well. In both cases, the exponential fit is probability $=a \exp (-$ entanglement $/ b)$. For $t=1.25$, we have $a$ $=0.01837, b=0.21543$, while for $t=20$, we have $a=0.00577, b$ $=0.150$ 53. Just like in Fig. 2, the "zero" entanglement zone is not shown in the figure (see caption of Fig. 2 and text). Also, including the zero entanglement probability, the curves are in fact correctly normalized.

neighbors, the same argument predicts that multipartite entanglement should be nonvanishing due to the fact that the volume of separable states is "super-doubly-exponentially small" with increasing number of parties [31].

\section{Dependence of nearest neighbor entanglement on the initial state}

Since we are dealing with a physical system that is governed by an Ising-interaction model, and as we want to generate as much entanglement as possible in the evolved state of the system, a natural choice of the initial state is a product state that will be created by a strong (initial) transverse field. Therefore to generate entanglement in a system governed by the Hamiltonian $H_{E-A}$, we have chosen the initial state as $\Pi_{i}|+\rangle_{i}$. This is also the inituition that we obtain from previous works on generation of entanglement in models with Ising-type interactions. See, e.g., $[17,18]$.

The generated entanglement does depend on the chosen initial state. In particular, any state of the form

$$
\prod_{i}\left|\psi_{i}^{z}\right\rangle
$$

where $\left|\psi_{i}^{z}\right\rangle$ is either $|0\rangle$ or $|1\rangle$ at the $i$ th lattice site, does not produce any entanglement.

The choice of product states as initial states is motivated by the fact that such states will be relatively easy to prepare in the laboratory. However, one may consider initial states 
that are entangled, and we consider here the following particular case. Consider a 1D lattice, and suppose that the initial state is

$$
|\mathrm{Bell}\rangle=\prod_{i}\left|\phi^{+}\right\rangle_{2 i-1,2 i},
$$

where $\left|\phi^{+}\right\rangle=\frac{1}{\sqrt{2}}(|00\rangle+|11\rangle)$.

As time goes by, the state of the two neighboring spins $2 i-1$ and $2 i$ is the rank-two state

$$
\begin{aligned}
\varrho_{2 i-1,2 i}^{\text {Bell }}\left(t,\left\{J_{i j}\right\}\right)= & \frac{1}{8}[4(|00\rangle\langle 00|+| 11\rangle\langle 11|)+(\cos 2 x+\cos 2 y) \\
& \times(|00\rangle\langle 11|+| 00\rangle\langle 11|)],
\end{aligned}
$$

where

$$
x=J_{2 i-2,2 i-1}+J_{2 i, 2 i+1}, \quad y=J_{2 i-2,2 i-1}-J_{2 i, 2 i+1} .
$$

Here we have assumed that the 1D lattice is either infinite, or it has a finite even number of sites. In the case of a finite lattice, we assume that the lattice is either with open boundary conditions, in which case the lattice must be at least of length 4 , and the pair $(2 i-1,2 i)$ must not be at the boundary, or the lattice is with periodic boundary conditions, in which case the lattice must be at least of length 6 . Simple calculations reveal that the time evolved state of the sites $2 i-1$ and $2 i$ is entangled at all times (in particular, its logarithmic negativity is positive). Notice that the sites $2 i-1$ and $2 i$ were initially in the state $\left|\phi^{+}\right\rangle$.

The evolved state of the sites $2 i$ and $2 i+1$ is different: it is separable at all times. Indeed, the state at all times is $\frac{1}{4} \mathbf{1}$ $\otimes 1$. Note that this is even before we perform any quenched averaging. Note also that the sites $2 i$ and $2 i+1$ were initially in the completely depolarized state.

Since the Hamiltonian commutes with the operator that is a tensor product of $\sigma^{z}$ at all or some lattice sites, the same above states are obtained for the initial state

$$
\prod_{i}\left|\phi^{-}\right\rangle_{2 i-1,2 i},
$$

where $\left|\phi^{-}\right\rangle=\frac{1}{\sqrt{2}}(|00\rangle-|11\rangle)$. However, even for the initial states

$$
\prod_{i}\left|\psi^{\ddagger}\right\rangle_{2 i-1,2 i},
$$

where $\left|\psi^{\ddagger}\right\rangle=\frac{1}{\sqrt{2}}\left(|01\rangle_{ \pm}|10\rangle\right)$, the same evolved states as above are obtained, up to local unitary operations. Indeed, the evolved state at the sites $2 i-1$ and $2 i$ is

$$
\begin{aligned}
\varrho_{2 i-1,2 i}^{\text {Bell }}\left(t,\left\{J_{i j}\right\}\right)= & \frac{1}{8}[4(|01\rangle\langle 01|+| 10\rangle\langle 10|)+(\cos 2 x+\cos 2 y) \\
& \times(|01\rangle\langle 10|+| 10\rangle\langle 01|)] .
\end{aligned}
$$

\section{IMPLEMENTATION OF QUANTUM GATES VIA DYNAMICS OF A SPIN GLASS SYSTEM}

Of the two questions posed in the Introduction, question (i), viz. "Can one generate entanglement in such systems that would survive quenched averaging over long times?" was answered affirmatively in the preceeding section, for the case of a disordered short range Hamiltonian. In this section, we consider the second question, viz. "Can one realize quantum gates with reasonable fidelity in disordered systems?" and show that for the same Hamiltonian [as in Eq. (1)], the answer is also positive.

We focus on the Hadamard gate, which transforms the computational basis into a complementary basis:

$$
\begin{gathered}
|0\rangle \rightarrow|+\rangle, \\
|1\rangle \rightarrow|-\rangle .
\end{gathered}
$$

We assume that the computation is performed in a spin lattice, of which particles 1 and 2 are part of and that at a certain time, particle 1 is in an arbitrary state $a|0\rangle+b|1\rangle$, where $|a|^{2}+|b|^{2}=1$, while particle 2 is in $|+\rangle$. Then we let particles 1 and 2 evolve according to the Hamiltonian $H_{E-A}$ for a suitable duration of time, before performing a measurement on particle 1 (in a suitable basis).

From the behavior of average entanglement in this model, one may infer that realization of quantum gates in such a model is not possible. However, we observe that this is not the case. For example, our results show that for the nonfrustrated case $\left(J=5, \sigma^{2}=1\right)$, particle 2 attains the Hadamard rotated state $a|+\rangle+b|-\rangle$, with quenched averaged fidelity greater than 0.85 . Moreover, one can increase such fidelity by increasing the number of spins and employing assisted measurements (choosing measurement bases depending on results of previous measurements). Note that the fidelity of the Hadamard rotated state using the classical information obtained from the measurement of particle 1 is only $2 / 3$ [32].

A similar system can potentially be used for implementing a two-qubit gate, say $U$. A possible way is to consider a square of four spin $1 / 2$ particles interacting via the Hamiltonian $H_{E-A}$ with $J=5, \sigma^{2}=1$, where, initially, two of the spins are in an arbitrary two-qubit state and the other two are each in the state $|+\rangle$. Similarly, as in the case of the Hadamard gate, it is conceivable that by suitably chosen measurements on two spins at a suitably chosen time, an approximately $U$-rotated two-qubit state of the initial two-qubit state will be obtained at the other two (unmeasured) spins.

\section{GENERATION OF ENTANGLEMENT IN A QUANTUM NEURAL NETWORK MODEL}

Consider now a long range Ising spin model, described by the Hamiltonian

$$
H_{l r}=\frac{c}{N} \sum_{i, j} J_{i j} \sigma_{i}^{z} \sigma_{j}^{z},
$$

where $N$ is the total number of spins. Again the factor $c$ helps one to make the couplings $J_{i j}$ dimensionless, and to make them effectively of the order of unity. We have used the same notation as in the short range Hamiltonian, but there is no room for confusion. Such models can be realized, e.g., with trapped ions [12] (cf. $[10,20]$ ), where the interactions, 


$$
J_{i j}=\sum_{\mu} \xi_{\mu}^{i} \xi_{\mu}^{j} / \lambda_{\mu}^{2},
$$

are phonon-mediated, with $\xi_{\mu}^{i}\left(\lambda_{\mu}\right)$ describing the phonon eigenmodes (eigenfrequencies). The (experimentally feasible) control of the external trap potential allows one to modify the value of the first eigenfrequencies. Here we consider two extreme cases. First, we take

$$
\begin{gathered}
\lambda_{1}=1, \quad \xi_{1}^{i}=\text { const } \forall i, \\
\lambda_{\mu} \rightarrow \infty \quad \text { for } \mu \geqslant 2,
\end{gathered}
$$

so that the interactions are ordered, and the Hamiltonian is

$$
H_{\text {lro }}=\frac{c}{N} S^{2},
$$

where $S=\sum_{i=1}^{N} \sigma_{i}^{z}$. Second, we consider the case when

$$
\lambda_{\mu}=1 \text { for all } \mu,
$$

when the Hamiltonian becomes

$$
H_{\text {neural }}=\frac{c}{N} \sum_{i, j=1}^{N} \sum_{\mu=1}^{p} \xi_{\mu}^{(i)} \xi_{\mu}^{(j)} \sigma_{i}^{z} \sigma_{j}^{z} .
$$

This latter one corresponds to the Hopfield model of a neural network with Hebbian couplings [14]. Here $p$ is the number of "patterns" stored by the neural network. The patterns are described by the random variables $\xi_{\mu}^{(i)}$, which take the values \pm 1 , with equal probabilities. To study the dynamics of entanglement for ordered and disordered Hamiltonians, we again take the initial state as $|\Psi\rangle=\prod_{i=1}^{N}|+\rangle_{i}$. We provide an efficient method to analytically compute the evolved state of any number of patterns and any number of spins.

\section{A. Behavior of entanglement for the ordered Hamiltonian}

Consider first the long range ordered Hamiltonian $H_{l r o}$. We write the evolution operator $\exp \left(-i S^{2} t / N\right)[27]$ as

$$
\int d \omega \exp \left[(i / N) \omega^{2}+S \sqrt{t}(-2 i / N) \omega\right]
$$

up to a constant factor, by going to the Fourier space. Applying now this unitary to the initial state $|\Psi\rangle$, we find any two-party state

$$
\varrho_{12}^{l r o}(t)=\operatorname{tr}_{k \neq 1,2} \rho^{l r o}(t)
$$

of such a system and compute the entanglement quantified by the LN. (This method can be also applied to find multipartite evolved states.) In Fig. 4, we plot the entanglement (as quantified by LN) of $\varrho_{12}^{l r o}(t)$, with respect to time, as well as $N$. The figure shows revivals of bipartite entanglement that occur on the time scale

$$
\tau_{R} \sim N \frac{\hbar}{c},
$$

and persist on the time scale (collapse time)

$$
\tau_{C} \sim \sqrt{N} \frac{\hbar}{c} .
$$

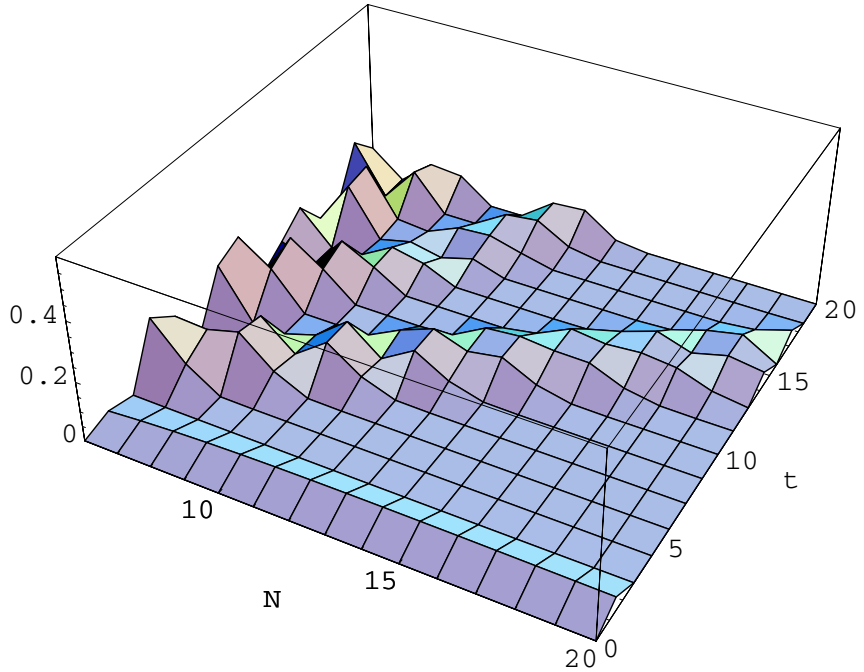

FIG. 4. (Color online) Generation of entanglement $\left[E_{L N}(t)\right.$, on the vertical axis] of bipartite states $\varrho_{12}^{\text {lro }}(t)$ with respect to time $t$ and number of spins $N$ [27]. Collapses and revivals of the entanglement are clearly depicted.

As depicted in Fig. 4, there are large ranges of time, for which the bipartite state is separable. Interestingly, this range of separability can be sharply reduced, considering entanglement of the tripartite evolved state $\rho_{123}^{\text {lro }}(t)$ in a bipartite cut. Although the interactions in $H_{l r o}$ are long range, they are ordered so that $\rho_{12}^{\text {lro }}(t)$ and $\rho_{123}^{\text {lro }}(t)$ take relatively simple forms.

\section{B. A disordered long range model: The neural network Hamiltonian}

Amazingly, the previous method applies also for $H_{\text {neural }}$, where the interactions are both long range and disordered. Despite its increased complexity, we can still use the technique for the evolution operator $\exp \left(-i H_{\text {neural }} t\right)[27]$ that was used in the case of $H_{\text {lro }}$. Specifically, we replace in $\exp \left(-i H_{\text {neural }} t\right)$ the operator $\exp \left(-i S_{\mu}^{2} t / N\right)$ by

$$
\int d \omega_{\mu} \exp \left[(i / N) \omega_{\mu}^{2}+S_{\mu} \sqrt{t}(-2 i / N) \omega_{\mu}\right]
$$

for every $\mu$, where $S_{\mu}=\sum_{i=1}^{N} \xi_{\mu}^{(i)} \sigma_{i}^{z}$. Applying this operator to our initial state $|\Psi\rangle$, we find that the $N$-particle state at time $t$ is given by

$$
\begin{aligned}
& \varrho^{\text {neural }}(t)=\int\left(\prod_{\mu} d r_{\mu} d s_{\mu}^{\prime}\right) e_{\mu}^{i \Sigma} r_{\mu} s_{\mu} / N \prod_{i=1}^{N}\left[e_{\mu}^{-2 i \sqrt{T} \Sigma \xi_{\mu}^{(i)} s_{\mu} / N}(|0\rangle\langle 0|)_{i}\right. \\
& +e^{2 i \sqrt{t} \Sigma} \xi_{\mu}^{(i)} s_{\mu} / N(|1\rangle\langle 1|)_{i} \\
& \left.+\left\{e^{-2 i \sqrt{t} \Sigma} \xi_{\mu}^{(i)} r_{\mu} / N(|0\rangle\langle 1|)_{i}+\text { H.c. }\right\}\right],
\end{aligned}
$$

where $r_{\mu}=\omega_{\mu}+\omega_{\mu}^{\prime}, s_{\mu}=\omega_{\mu}-\omega_{\mu}^{\prime}$, with $\mu=1, \ldots, p$. After tracing out over all particles except 1 and 2 , we obtain 


$$
\begin{aligned}
\varrho_{12}^{\text {neural }}(t)= & \frac{1}{4} \mathbf{1} \otimes \mathbf{1}+\frac{1}{4}\left(\left\{e_{\mu}^{-4 i t \Sigma} \xi_{\mu}^{(1)} \xi_{\mu}^{(2)} / N\left[\prod_{i \neq 1,2} \cos \left(4 t \sum_{\mu} \xi_{\mu}^{(i)} \xi_{\mu}^{(2)} / N\right)|00\rangle\left\langle 01\left|+\prod_{i \neq 1,2} \cos \left(4 t \sum_{\mu} \xi_{\mu}^{(i)} \xi_{\mu}^{(1)} / N\right)\right| 00\right\rangle\langle 10|\right]\right\}\right. \\
& +\prod_{i \neq 1,2} \cos \left(4 t \sum_{\mu} \xi_{\mu}^{(i)}\left(\xi_{\mu}^{(1)}+\xi_{\mu}^{(2)}\right) / N\right)|00\rangle\left\langle 11\left|+\prod_{i \neq 1,2} \cos \left(4 t \sum_{\mu} \xi_{\mu}^{(i)}\left(\xi_{\mu}^{(1)}-\xi_{\mu}^{(2)}\right) / N\right)\right| 01\right\rangle\langle 10| \\
& \left.+\left\{e_{\mu}^{4 i t \sum \xi_{\mu}^{(1)} \xi_{\mu}^{(2)} / N}\left[\prod_{i \neq 1,2} \cos \left(4 t \sum_{\mu} \xi_{\mu}^{(i)} \xi_{\mu}^{(1)} / N\right)|01\rangle\left\langle 11\left|+\prod_{i \neq 1,2} \cos \left(4 t \sum_{\mu} \xi_{\mu}^{(i)} \xi_{\mu}^{(2)} / N\right)\right| 10\right\rangle\langle 11|\right]\right\}+\text { H.c. }\right) .
\end{aligned}
$$

For $N$ large, and $t / N$ small, the above expression can be simplified using the fact that, for example,

$$
\begin{aligned}
& \left|\prod_{i \neq 1,2} \cos \left(4 t \sum_{\mu} \xi_{\mu}^{(i)} \xi_{\mu}^{(2)} / N\right)\right| \\
& \quad=\exp \left[\sum_{i \neq 1,2} \log _{e}\left|\cos \left(4 t \sum_{\mu} \xi_{\mu}^{(i)} \xi_{\mu}^{(2)} / N\right)\right|\right] \\
& \quad \approx \exp \left[-\left(8 t^{2} / N^{2}\right) \sum_{i \neq 1,2}\left(\sum_{\mu} x_{\mu}^{i}\right)^{2}\right],
\end{aligned}
$$

where for all $i, x_{\mu}^{i}=+1$ or -1 with probability $1 / 2$ each. Therefore for large $N$ and small $t / N$, we have that

$$
\prod_{i \neq 1,2} \cos \left(4 t \sum_{\mu} \xi_{\mu}^{(i)} \xi_{\mu}^{(2)} / N\right)
$$

self-averages (i.e., independent of a particular disorder realization) to the value $\exp \left[-\left(8 t^{2} p / N\right)\right]$ (see [33]), so that after time $t \sim \sqrt{N / p}$, all the off-diagonal elements of the state $\varrho_{12}^{\text {neural }}(t)$ become vanishingly small. Therefore nearest neighbor entanglement in the evolved state appears and persists for times of order

$$
\tau_{C} \sim \sqrt{\frac{N}{p}} \frac{\hbar}{c}
$$

There are repeated revivals in entanglement, with the period being [34]

$$
\begin{gathered}
\tau_{R}=\frac{\pi N}{2} \frac{\hbar}{c} \quad \text { for odd } p, \\
\tau_{R}=\frac{\pi N}{4} \frac{\hbar}{c} \text { for even } p .
\end{gathered}
$$

Note that the period of revivals is independent of the number of patterns in the model (cf. [25]).
It is to be noted here that in the case of short range models considered in preceeding sections, although the entanglement persists at long times, there are no collapse-revivals. Here, however, the long range interactions force the entanglement to continue exhibiting (repeated) collapse-revivals, even at long times.

\section{SUMMARY}

Summarizing, we have studied entanglement of disordered and complex quantum spin systems with short range and long range interactions that can be realized with trapped atoms or ions. We have shown that in both cases, it is possible to generate quenched averaged entanglement over long times. In the case of short range interactions, we have considered the Edwards-Anderson model in several lattices. The cases of 1D and 2D square lattices are considered in detail. We have shown that in such disordered systems, it is possible to implement also distinctly quantum single-qubit gates with high fidelity. We provide an efficient analytic method to compute the time evolution of a state in a system with long range interactions. By using this method, we are able to demonstrate the generation of entanglement in spin systems with long range interactions, e.g., in the Hopfield neural network model. We have shown that in such case, entanglement exhibits a sequence of collapses and revivals.

\section{ACKNOWLEDGMENTS}

We thank I. Bloch, H.-P. Büchler, J. Eschner, M. Pons, L. Sanchez-Palencia, J. Wehr, and P. Zoller for fruitful discussions. We acknowledge support from the DFG (SFB 407, SPP1078, SPP1116, and 436POL), Spanish MEC grants BFM-2002-02588, MEC Consolider Project QOIT, FIS2005-04627, the Alexander von Humboldt Foundation, the ESF Program QUDEDIS, and EU IP SCALA.
[1] The Physics of Quantum Information, edited by D. Bouwmeester, A. Ekert, and A. Zeilinger (Springer, Berlin, 2000).

[2] J. I. Cirac and P. Zoller, Phys. Rev. Lett. 74, 4091 (1995).

[3] F. Schmidt-Kaler, H. Häffner, M. Riebe, S. Gulde, G. P. T. Lancaster, T. Deuschle, C. Becher, C. F. Roos, J. Eschner, and R. Blatt, Nature (London) 422, 408 (2003); J. Chiaverini, D. Leibfried, T. Schaetz, M. D. Barrett, R. B. Blakestad, J. Britton, W. M. Itano, J. D. Jost, E. Knill, C. Langer, R. Ozeri, and D. J. Wineland, ibid. 432, 602 (2004); C. F. Roos, M. Riebe, H. Haffner, W. Hansel, J. Benhelm, G. P. T. Lancaster, C. Becher, F. Schmidt-Kaler, and R. Blatt, Science 304, 1478 (2004); P. Walther, K. J. Resch, T. Rudolph, E. Schenck, H. Weinfurter, V. Vedral, M. Aspelmeyer, and A. Zeilinger, Nature (London) 434, 169 (2005).

[4] G. K. Brennen, C. M. Caves, P. S. Jessen, and I. H. Deutsch, Phys. Rev. Lett. 82, 1060 (1999); D. Jaksch, J. I. Cirac, P. 
Zoller, S. L. Rolston, R. Cote, and M. D. Lukin, ibid. 85, 2208 (2000); O. Mandel, M. Greiner, A. Widera, T. Rom, T. W. Hänsch, and I. Bloch, Nature (London) 425, 937 (2003).

[5] P. Rabl, A. J. Daley, P. O. Fedichev, J. I. Cirac, and P. Zoller, Phys. Rev. Lett. 91, 110403 (2003).

[6] J. J. Garcia-Ripoll and J. I. Cirac, Phys. Rev. Lett. 90, 127902 (2003).

[7] B. Damski, J. Zakrzewski, L. Santos, P. Zoller, and M. Lewenstein, Phys. Rev. Lett. 91, 080403 (2003).

[8] A. Sanpera, A. Kantian, L. Sanchez-Palencia, J. Zakrzewski, and M. Lewenstein, Phys. Rev. Lett. 93, 040401 (2004).

[9] M. Mézard, G. Parisi, and M. A. Virasoro, Spin Glass and Beyond (World Scientific, Singapore, 1987).

[10] D. Porras and J. I. Cirac, Phys. Rev. Lett. 92, 207901 (2004).

[11] For a review see, M. Baranov, Ł. Dobrek, K. Goral, L. Santos, and M. Lewenstein, Phys. Scr., T T102, 74 (2002); for the first experiment see, P. O. Schmidt, S. Hensler, J. Werner, A. Griesmaier, A. Gorlitz, T. Pfau, and A. Simoni, Phys. Rev. Lett. 91, 193201 (2003).

[12] M. Pons, V. Ahufinger, C. Wunderlich, A. Sanpera, and M. Lewenstein, e-print cond-mat/0512606; M. Pons, V. Ahufinger, C. Wunderlich, A. Sanpera, S. Braungardt, A. Sen(De), U. Sen, and M. Lewenstein, e-print cond-mat/0607016.

[13] H. Cho, T. D. Ladd, J. Baugh, D. G. Cory, and C. Ramanathan, Phys. Rev. B 72, 054427 (2005).

[14] D. J. Amit, Modeling Brain Function (Cambridge University Press, Cambridge, England 1992).

[15] P. W. Shor, Phys. Rev. A 52, R2493 (1995); A. M. Steane, Proc. R. Soc. London, Ser. A 452, 2551 (1996).

[16] M. Lewenstein, L. Santos, M. A. Baranov, and H. Fehrmann, Phys. Rev. Lett. 92, 050401 (2004).

[17] H. J. Briegel and R. Raussendorf, Phys. Rev. Lett. 86, 910 (2001); R. Raussendorf and H. J. Briegel, ibid. 86, 5188 (2001); R. Raussendorf, D. E. Browne, and H. J. Briegel, Phys. Rev. A 68, 022312 (2003).

[18] W. Dür, L. Hartmann, M. Hein, M. Lewenstein, and H.-J. Briegel, Phys. Rev. Lett. 94, 097203 (2005).

[19] G. Vidal and R. F. Werner, Phys. Rev. A 65, 032314 (2002).

[20] F. Mintert and C. Wunderlich, Phys. Rev. Lett. 87, 257904 (2001)

[21] J. H. Eberly, N. B. Narozhny, and J. J. Sanchez-Mondragon, Phys. Rev. Lett. 44, 1323 (1980).

[22] A. Peres, Phys. Rev. Lett. 77, 1413 (1996); M. Horodecki, P. Horodecki, and R. Horodecki, Phys. Lett. A 223, 1 (1996).

[23] M. Horodecki, P. Horodecki, and R. Horodecki, Phys. Rev. Lett. 78, 574 (1997).

[24] J. Calsamiglia, L. Hartmann, W. Dür, and H. J. Briegel, Phys.
Rev. Lett. 95, 180502 (2005).

[25] L. Hartmann, J. Calsamiglia, W. Dür, and H. J. Briegel, Phys. Rev. A 72, 052107 (2005).

[26] Suppose, for example, that for a fixed $i$ and $j, J_{i j}$ is Gaussian distributed, with mean $\mu$, and variance $s^{2}$. Consequently, $a J_{i j}$ $+b$ will be Gaussian distributed with mean $a \mu+b$, and variance $(a \varsigma)^{2}$, where $a$ and $b$ are real numbers. So, for large $t$, $J_{i j} t / 2$ will be a Gaussian distribution with a very large variance, so that near the mean, the distribution is approximately uniform. Due to the fact that $J_{i j} t / 2$ always appears as arguments of trigonometric functions, the distribution can be approximated by a uniform distribution over $[0,2 \pi]$.

[27] We remind the reader that $t$ is implicitly multiplied by $c / \hbar$, where $c$ is the multiplicative constant in the relevant Hamiltonian.

[28] K. Życzkowski, P. Horodecki, A. Sanpera, and M. Lewenstein, Phys. Rev. A 58, 883 (1998).

[29] The summation in Eq. (5) runs over $d$ terms. This is under the assumption that the lattice under consideration is such that there does not appear a situation where a single lattice site is a neighbor to both 1 and 2, where 1 and 2 are an arbitrary pair of neighboring lattice sites. This, e.g., happens for the linear chain, the $2 \mathrm{D}$ honeycomb lattice, the $2 \mathrm{D}$ square, and the $3 \mathrm{D}$ cube. In cases where this does not happen, a prominent example being the triangular lattice, the qualitative features would presumably remain the same.

[30] Note that in the relevant two qubit states, the $\phi_{k}=J_{i j} t / 2$ 's always appear inside cosines, so that the period is really $\pi$, and not $2 \pi$.

[31] S. I. Szarek, Phys. Rev. A 72, 032304 (2005).

[32] S. Popescu, Phys. Rev. Lett. 72, 797 (1994).

[33] To see this, note that each $X^{i}=\Sigma_{\mu}^{p} x_{\mu}^{i}$ is binomially distributed (up to a shift), taking up the values $-p,-p+2, \ldots, p$, which correspond, respectively, to $0,1, \ldots, p$ "successes," where a "success" is the appearance of the +1 value of $x_{\mu}^{i}$ for some $\mu$ in the sum $\Sigma_{\mu}^{p} x_{\mu}^{i}$. The probability that $X^{i}$ takes up the value $-p+2 r$ (corresponding to $r$ successes, $r=0,1, \ldots, p)$ is $P[\#$ of successes $=r]=\frac{p !}{r !(p-r) !}\left(\frac{1}{2}\right)^{p}$. Note also that in the subsequent sum $\sum_{i \neq 1,2}\left(\sum_{\mu}^{p} x_{\mu}^{i}\right)^{2}=\sum_{i \neq 1,2}\left(X^{i}\right)^{2}$, the probability of $X^{i}$ taking the value $(-p+2 r)^{2}$ is $P$ [\# of successes $\left.=r\right]$ $+P[\#$ of successes $=p-2 r]$.

[34] To see this, take, for example, the coefficient of $|10\rangle\langle 11|$ in Eq. (15): $\Sigma_{\mu=1}^{p} \xi_{\mu}^{(i)} \xi_{\mu}^{(2)}$ takes the values $-p,-p+2, \ldots, p$. These values are either all even or all odd (depending on whether $p$ is even or odd). This dictates the periodicity of entanglement in the state. 